\title{
Mst1 Inhibition As A Cellular Mediator: Prevention Of Anoikis In mBMSCs Through Activating ITGa5 $31 / F A K$ Signaling Pathway
}

\section{Tao Zhang}

Shandong Provincial Hospital

Qian Zhang

Shandong Provincial Hospital

Shang Wu

Shandong Provincial Hospital

Haizhou Zhang

Shandong Provincial Hospital

Wancheng Yu ( $\nabla$ yuwancheng123@126.com )

Shandong Provincial Hospital https://orcid.org/0000-0003-3850-3298

\section{Research Article}

Keywords: mouse bone marrow mesenchymal stem cell, Mst1, anoikis, integrin, focal adhesion kinase

Posted Date: October 25th, 2021

DOI: https://doi.org/10.21203/rs.3.rs-977461/v1

License: (c) (i) This work is licensed under a Creative Commons Attribution 4.0 International License. Read Full License 


\section{Abstract}

BACKGROUND: Lost of adhesion of the mesenchymal stem cell (MSC) to the extracellular matrix (i.e., anoikis) caused poor survival, which limited the therapeutic efficacy of the MSCs. We recently found that the suppression of the mammalian sterile 20-like kinase 1 (Mst1) protected the mouse bone marrow MSCs (mBMSCs) from ROS-mediated cell apoptosis. However, the effect of Mst1 inhibition on the anoikis of $\mathrm{mBMSC}$ remains elusive.

METHODS: We propose the use of poly-HEMA-induced anoikis following the Mst1 silencing in the mBMSCs, then cell anoikis were tested by Anoikis Assay Kit and TUNEL Assay Kit, and the change of integrin (ITG)/FAK signal pathway were measured.

RESULTS: The mBMSCs were susceptible to anoikis but became anoikis-resistant via Mst1 inhibition. In addition, $\mathrm{p}$ - focal adhesion kinase (p-FAK) was upregulated, whereas activated caspase 3 was downregulated in the poly-HEMA-treated mBMSC/sh-Mst1. Mst1 inhibition activated ITGa5 and ITG $\beta 1$ but not ITGa4, ITGav, or ITGß3. Further analysis revealed that anoikis resistance conferred by sh-Mst1 was disrupted by either inhibiting ITGa5 or ITG $\beta 1$ by using siRNA.

CONCLUSIONS: Mst1 inhibition resulted in the anoikis resistance of mBMSCs by activating the ITGa5 $31 /$ FAK signaling pathway. Inactivation of Mst 1 may provide a promising strategy to overcome the anoikis of the implanted MSCs.

\section{Introduction}

Mesenchymal stem cell (MSC) therapy offers an attractive therapeutic approach for pulmonary arterial hypertension (PAH) because of their antiinflammatory, immunomodulatory, and regenerative properties [1]. The therapeutic potential of MSC therapy to improve disease-associated parameters and the underlying encouraging outcomes for PAH have been demonstrated $[2,3]$. However, less than $5 \%$ of the transplanted cells are retained in vivo [4]. Therefore, the low survival rate is likely to be the major obstacle to a therapeutic MSC-based approach to PAH.

Anoikis is a form of programmed cell death when the cells were detached from the extracellular matrix (ECM), resulting in apoptosis due to the loss of integrin (ITG)-mediated signals [5]. ITGs are transmembrane molecules that exist as an a: $\beta$ heterodimeric complex, which play a role in cell survival [6, 7]. Studies have shown that ITGs have different abilities to protect cells from anoikis by utilizing diverse signaling pathway, including focal adhesion kinase (FAK) signal [5]. Upon detachment, FAK is one of the key ITG signal molecules recruited into the focal adhesion sites, consequently activating the cell survival signal. MSCs have similar features with tumor cells in the ability of unlimited reproduction and some common molecular pathways $[8,9]$. Moreover, substantial evidence has indicated that anoikis is potentially a major contributor to the apoptosis of transplanted cells [10,11]. After detachment from the culture dish for engraftment and injection into the damaged tissue/organ, MSCs encounter the lack of matrix support and adhesion to ECM. This condition leads to a low propensity to the proliferation and 
adhesion and a change in the series of anoikis signaling pathways $[3,12,13]$. Thus, accumulating studies have suggested that the regulation of molecules involving adhesion and apoptosis may decrease the anoikis of the transplanted MSCs $[14,15]$.

We have previously demonstrated that inhibition of the mammalian sterile 20-like kinase 1 (Mst1) improved the cell viability in $\mathrm{H}_{2} \mathrm{O}_{2}$-induced mouse bone marrow mesenchymal stem cells (mBMSCs) [16]. Mst1 (also known as Stk4) is a serine-threonine kinase that constitutes a key component of the Hippo signaling pathway, regulates cell apoptosis, and interacts with ITG [17]. However, the effect of Mst1 inhibition on the anoikis, especially the signal pathway controlled by Mst1 inhibition in the anoikis of mBMSCs, has not been clearly defined.

In this study, we examined the potential role of Mst1 inhibition in the regulation of anoikis of mBMSCs and investigated the change in the ITG/FAK signal by Mst1 inhibition.

\section{Methods}

\section{Cell culture}

The mBMSCs were obtained as previously described [16]. Cultured mBM-MSCs between passages 3 and 5 were used for the following experiments.

\section{Adenovirus infection}

The adenovirus harboring Mst1 shRNA (Ad-sh-Mst1) and the control vector for Mst1 shRNA (Ad-NC-Mst1) were purchased from WZ Biosciences (China). Vector details have been described previously [16]. The shRNA sequence targeting mouse Mst1 was GCCCTCACGTA GTCAAGTATT.

\section{Nude mouse tumorigenicity}

A total of 10 female nude mice (4 weeks old) were purchased from the Beijing Vital River Laboratory Animal Technology Co., Ltd. (No. 110011211101205428) and maintained in a specific pathogen-free environment. Then, $5.0 \times 10^{6}$ of the mBMSCs, mBMSC/NC-Mst1, and mBMSC/sh-Mst1 were injected into the right flank near the hind leg of each nude mouse. The tumors were measured with a caliper every 4 days. At 60 days after cell inoculation, all mice were terminated with ether anesthesia, and the tissues were harvested. All animal procedures were approved by the Animal Care and Use Committee of Shandong First Medical University (No. 2021-177).

\section{Differentiation capacity assay}

For the osteogenic differentiation, mBMSCs, mBMSC/NC-Mst1, and mBMSC/sh-Mst1 were cultured for 21 days in a complete a-MEM medium containing $10^{-7} \mathrm{M}$ dexamethasone, $10 \mathrm{mM} \beta$-glycerol phosphate, and $50 \mu \mathrm{M}$ ascorbate-2-phosphate. The media were changed every 3 days. Calcium deposits were detected by alkaline phosphatase staining. 
For the adipogenic differentiation, the cells were incubated for 21 days in a complete a-MEM medium containing $10^{-6} \mathrm{M}$ dexamethasone, $0.5 \mu \mathrm{M}$ isobutylmethylxanthine, and $10 \mathrm{ng} / \mathrm{ml}$ insulin. The adipogenic induction medium was changed every 3 days. The lipoid substances were stained with Oil Red O. Both alkaline and Oil Red 0 staining were visualized by light microscopy.

\section{siRNA transfection}

The siRNAs were obtained from GenePharma (China). The sequences of the sense and antisense strands of the siRNA were as follows: mouse siRNA-ITGa5, 5》-GCAGGGAGAUGAAGAUCUACC》 (sense) and 5》UAGAUCUUCAUCUCCCUGCAG (antisense); mouse siRNA-ITG 31 , 5》-GGAGAACCACAGAAGUUUACA$3 \rrbracket$ (sense) and 5区-UAAACUUCUGUGGUUCUCCUG-3区 (antisense); and siRNA-negative control (NC), 5》UUCUCCGAACGUGUCACGUTT (sense) and 5囚-ACGUGACACGUUCGGAGAATT-3囚 (antisense). Then, $24 \mathrm{~h}$ after infecting Ad-sh-Mst1, the mBMSCs at 75\% confluence were transfected with ITGa5 or ITG $\beta 1$ or NC siRNA ( $50 \mathrm{nM}$ ) by using the Lipofectamine RNAi MAX (Invitrogen) according to the manufacturer's instructions. The level of ITGa5 or ITG $\beta 1$ expression was significantly blocked by the transfected siRNA.

\section{Cell treatment}

Dishes coated with poly-hydroxyethyl methacrylate (Poly-HEMA; Sigma, USA) were used to prevent the adhesion of cells to the tissue culture plates. Briefly, poly-HEMA stock were dissolved in $95 \%$ ethanol at a concentration of $12 \mathrm{mg} / \mathrm{ml}$, and $1 \mathrm{ml}$ of the $12 \mathrm{mg} / \mathrm{ml}$ poly-HEMA was added to each well of a 6-well plate, which was dried overnight in a clean bench. Cells were transfected as previously described. Cells (5 $\times 10^{5}$ ) were plated in each well coated with $12.5 \mathrm{mg} / \mathrm{ml}$ Poly-HEMA for a certain time.

\section{Cell viability assay}

The CCK-8 assay (Beyotime Institute of Biotechnology, China) was then employed to assess the cell viability. After being cultured in poly-HEMA-coated dishes, the cells were collected and seeded $\left(3 \times 10^{3} /\right.$ well) in 96 -well plates. Then, $10 \mu \mathrm{l} /$ well CCK-8 at $10 \%$ dilution was added to each well, and the plate was incubated for $1 \mathrm{~h}$ in an incubator. Spectrophotometric readings were normalized using cell-free medium as a blank. Absorbance was measured at $450 \mathrm{~nm}$ by using a Multiskan MK3 microplate reader, and the experiments were repeated thrice. Cell viability was calculated as the ratio of control.

\section{Cell adhesion}

After being cultured in poly-HEMA-coated dishes, the collected cells were resuspended in the complete aMEM medium and then plated in triplicates $\left(5 \times 10^{4}\right.$ cells/well) onto the well coated with fibronectin (10 $\mathrm{g} / \mathrm{ml}$ ), which was previously blocked for $1 \mathrm{~h}$ with $1 \%$ BSA. After $6 \mathrm{~h}$, the cells were washed by PBS and stained with crystal violet. Before $10 \%$ acetic acid was used, the unbound dye was removed by PBS. The absorbance was read at $630 \mathrm{~nm}$ by using a Multiskan MK3 microplate reader. The experiment was repeated thrice. Cell viability was calculated as the ratio of control.

\section{Anoikis assay}


Anoikis assay was performed using the Anoikis Assay Kit (ab211153, Abcam, USA) according to the manufacturer's instructions. After transfection, the cells were added to each well of the 96-well Anchorage Resistant Plate for $36 \mathrm{~h}$ and then treated with s AM/EthD-1. Quantitative measurement was performed using a microplate reader at Ex/Em = 485/515 nm (Calcein AM) and Ex/Em = 525/590 nm (EthD-1).

\section{Flow cytometry}

After $36 \mathrm{~h}$ of incubation in poly-HEMA-coated dishes, the cells were collected after centrifugation at 300 $\times g$ for 5 min and incubation in the antibody (ITGa4 [1/500 dilution, 553157, BD], ITGa5 [1/500 dilution, 557447, BD], ITGav [1/300 dilution, 740946, BD], ITGß1 [1/500 dilution, 561796, BD], ITGß3 [1/100 dilution, 740677, BD]) for $1 \mathrm{~h}$, which was performed according to the operation manual.

Anoikis was also analyzed using in situ Direct DNA Fragmentation (TUNEL) Assay Kit (ab66108, Abcam). After being cultured in poly-HEMA-coated plate, the cells were collected and added to $70 \%$ ice ethanol for 30 min. Then, ethanol was removed, and the cells were resuspended by a wash buffer. Then, the cells were stained with a staining solution for $60 \mathrm{~min}$. Prior to the addition of the PI/ RNase A solution, the cells were treated using a rinse buffer twice. Quantification was analyzed by the BD FACSDiva software $(\mathrm{Ex} / \mathrm{Em}=488 / 520 \mathrm{~nm}$ for FITC, and 488/623 $\mathrm{nm}$ for $\mathrm{PI})$.

\section{Quantitative PCR (qPCR)}

The qPCR was performed as previously reported [16]. The total mRNA from the mBMSCs underwent differentiation via 21-day exposure to osteogenic or adipogenic condition, or the cells transfected with siRNA were isolated using Trizol Reagent (Thermo Fisher Scientific, Inc.). The RNA was subsequently reverse transcribed to cDNA, and amplified using SYBR® Premix Ex Taq ${ }^{\mathrm{TM}} \otimes \mathrm{kit}$ (Takara, Dalian, China) on ABI 7500 real-time PCR system (Applied Biosystems). Each experiment was repeated thrice. Data were normalized using the housekeeping gene GAPDH by the $2^{-\triangle \triangle C T}$ method. The primer sequences are shown in the Supporting Information Table 1.

\section{Western blot analysis}

To determine the protein expression, Western blot analysis was performed. After being cultured in polyHEMA-coated plate, the whole-cell protein extracts were prepared in RIPA lysis buffer, subjected to SDSPAGE, and transferred onto PVDF membranes. The membranes were then blocked for $1 \mathrm{~h}$ with $5 \%$ skimmed milk or BSA in TBST and incubated overnight at $4{ }^{\circ} \mathrm{C}$ with the following primary antibodies (diluted by Western Primary Antibody Buffer, Beyotime): Mst1 (1:1000, ab51134, Abcam), ITGa5 (1:1000, ab150361, Abcam), ITGß1(1:1000, ab179471, Abcam), Phospho-FAK (Tyr397) (1:500, 3283S, CST), FAK $(1: 1,000,3285 S, C S T)$, activate caspase 3 (1:1000, ab214430, Abcam), and caspase 3 (1:1000, ab18297, Abcam). GAPDH (1:1000, 5174S, CST) served as the loading control. The anti-rabbit IgG and HRP-linked antibody $(1: 1000,7074 S, C S T)$ were used. The relative protein expression levels were compared with those of GAPDH by using ImageJ software.

\section{Statistical analysis}


All results were expressed as means \pm SD. The data were analyzed by one-way ANOVA. $P$ value less than 0.05 was considered significant.

\section{Results}

\section{Lost cell-ECM adhesion enhanced the aberrant apoptosis and Mst1 expression of the mBMSCs}

Poly-HEMA is used to construct an artificial anchorage-independent culture condition to mimic the suspended state of mBMSCs. Here, we assessed the susceptibility of mBMSCs to anoikis in detached condition. The results from the anoikis assay by TUNEL and Anoikis Assay Kit showed that the rate of mBMSC apoptosis increased in the detached-condition induced by poly-HEMA in a time-dependent manner (Figs. 1A-C). These results suggested that culturing in precoated Poly-HEMA plates induced anoikis of mBMSCs.

With the use of CCK-8, the cell proliferation of the mBMSCs also decreased in a time-dependent manner (Fig. 1D), and the mBMSCs exhibited decreased adhesion in the precoated poly-HEMA plates for 24,36 , and $48 \mathrm{~h}$ (Fig. 1E) compared with that at $0 \mathrm{~h}$. The ability of cell adhesion decreased after the cells were cultured in precoated poly-HEMA plates. These observations suggested that the anchorage-independent growth in mBMSCs were suppressed.

To determine the change in the Mst1 expression under the detached condition, we checked the expression level of the Mst1 proteins. The results indicated the upregulation of proapoptotic protein Mst1 in the detached mBMSCs (Fig. 1F), and Mst1 expression increased significantly after the cells were cultured for $36 \mathrm{~h}$ in the detached condition (Fig. 1F). These phenomena suggested that the anoikis mBMSCs exhibited higher expression of Mst1. Combined with the results in our previous study, Mst1 inhibition may shift the anoikis induced by poly-HEMA. Thus, Mst1-depleted mBMSCs were then examined to test the hypothesis that Mst1 inhibition protected the mBMSCs from anoikis. The mBMSCs were infected with the adenovirus harboring the Mst1 shRNA, and the mRNA and protein expression levels of Mst1 were analyzed by qPCR and Western blot (Supporting Information Fig. S1).

\section{The mBMSCs/sh-Mst1 exhibited multipotent capabilities in vitro and no oncogenesis in vivo}

We evaluated the effect of Mst1 inhibition on the osteogenic and adipogenic differentiation of the mBMSCs. As shown in Figs. 2A and C, the suppression of Mst1 was correlated with an increase in osteogenic and adipogenic differentiation of the mBMSCs. Then, to determine accurately the effects of Mst1 inhibition on the progression of osteogenic and adipogenic differentiation in the mBMSCs, qPCR was performed. Runx2 and Alkaline phosphatase (ALP), which are the osteoblast differentiation markers, were both significantly increased in the mBMSC/sh-Mst1 (Fig. 2B) [18]. In addition, in the adipogenic differentiation of mBMSCs, the mRNA level of the proliferator-activated receptor gamma (PPARg), a key 
regulator of adipogenic differentiation, was significantly elevated by Mst1 inhibition. The expression of the late adipogenesis marker, i.e., fatty acid binding protein 4 (FABP4), was also increased (Fig. 2D) [19].

No tumor-like mass appeared at 60 days postinjection in animals injected with mBMSC/sh-Mst1

(Fig. 2E). At 60 days postinjection, subcutaneous tissue, lung, liver, kidney and heart were harvested and weighed, and the weight of the lung, liver, kidneys and heart in each group were kept approximately the same (Fig. 2F and Supporting Information Table 2). Histological analysis showed no formation of stromal structures by the mBMSC/sh-Mst1 in the subcutaneous tissue (Fig. 2G).

\section{Suppression of Mst1 protected the mBMSCs from anoikis}

When combined with the change in anoikis, cell proliferation, cell adhesion, and Mst1 expression, the mBMSCs were cultured in precoated poly-HEMA plates for $36 \mathrm{~h}$. Anoikis-induced cell apoptosis of mBMSCs was detected using TUNEL and a commercial kit, in which the culture plate was coated with poly-HEMA, and Calcein AM/EthD-1 was used for anoikis detection. A significantly decreased cell death was observed in the mBMSCs/sh-Mst1 (Figs. 3A-C). Similar to the abovementioned, the ability of cell proliferation and adhesion were also improved by Mst1 inhibition (Fig. 3D, E). These results suggested that Mst1 inhibition significantly attenuated the ECM detachment-induced anoikis in the mBMSCs.

The immunoblot data further supported the above notion. FAK, a focal adhesion protein, is a main mediator of cell-substrate adhesion. Consistent with the increased ability of cell adhesion, mBMSC/shMst1 exhibited a robust FAK activation in Western blot analyses (Figs. 3F, G). Similar to apoptosis, anoikis is mediated by caspase activation. In this study, the effect of Mst1 inhibition on the activation of caspase 3 was measured using Western blot. As shown in Figs. 3F and G, Mst1 inhibition significantly suppressed the activation of caspase 3 in the suspension-grown mBMSCs. These data indicated that Mst1 inhibition reactivated the FAK/Caspase3 pathway in the anchorage-independent mBMSCs.

\section{Activation of ITGa5 $\beta 1$ by Mst1 inhibition conferred anoikis resistance to the mBMSCs}

Given that cell anoikis is mediated by the heterodimeric cell surface receptor ITG, we explored the role of ITGs in anoikis-resistant mBMSCs/sh-Mst1. First, we compared the expression profile of ITGa5, ITGav, ITGa4, ITG 31 , and ITG $\beta 3$ in the poly-HEMA-treated mBMSCs by flow cytometry. The levels of ITGa5, ITGav, ITGa4, ITG $\beta 1$, and ITG $\beta 3$ decreased significantly in the anoikis groups than in the control groups (Figs. 4A, B). The poly-HEMA-treated mBMSC/sh-Mst1 showed an increasing trend for ITGa5 and ITG $\beta 1$ compared with the anoikis mBMSCs, whereas the level of ITGav, ITGa4, and ITG $\beta 3$ showed no different expression profiles between the anoikis mBMSCs and poly-HEMA-treated mBMSCs/sh-Mst1 (Fig. 4A, B). These data indicated that the sh-Mst1 inhibition reactivated ITGa5 and ITG $\beta 1$.

To demonstrate the changes in ITGa5 and ITG $\beta 1$, we studied their role in anoikis-resistant mBMSCs/shMst1 as our model. We performed the anoikis susceptibility by their individual genetic ablation. The transfection efficiencies are shown in Figs. 4C-F. In the anoikis-resistant mBMSC/sh-Mst1, the siRNAmediated ablation of ITGa5 or ITG $\beta 1$ reversed cell death, cell proliferation, and cell adhesion (Figs. 5A-E), 
and the FAK/Caspase 3 pathway was inactivated by either ITGa5 or ITG $\beta 1$ siRNA (Figs. 5F-K). Knockdown of ITGa5 or ITG $\beta 1$ did not affect the expression of Mst1 (Fig. 5G).

\section{Discussion}

Compelling evidence has indicated that anoikis limits the therapeutic efficacy of MSCs implanted for tissue repair [20]. Here, we provided the first demonstration that mBMSC/sh-Mst1 could survive after the detachment from the substratum by the process called anoikis resistance. We also showed that this response was mediated by the upregulation of the ITGa5 $\beta 1 / F A K$ signal.

Loss of cell-ECM adhesion promotes death by anoikis, the perturbation of which leads to abnormal cell proliferation [21]. By contrast, enhancing the adhesion of MSCs may benefit the MSC-based therapies that exploit the tropisms of MSC for the injured sites by guiding permanent anchorage to the target tissue $[22,23]$. Moreover, the acquisition of anoikis resistance could facilitate anchorage-independent growth [24]. In the poly-HEMA-treated mBMSC systems, we noticed that upon detachment, the mBMSCs underwent apoptosis and loss of cell proliferation and cell adhesion. However, combined with the previous study, Mst1 inhibition not only overcame anchorage-dependent apoptosis (i.e., anoikis) of the mBMSCs but also improved mBMSC adhesion to ECM and cell proliferation. Therefore, Mst1 inhibition may enhance the survival of the transplanted mBMSCs through the inhibition of anoikis.

Mst1 has been demonstrated to serve as a key component of a multistep signaling pathway that controls various cellular processes, including cell differentiation. [25]. In the present study, we showed that Mst1 inhibition improved the osteogenic and adipogenic differentiation capability of mBMSCs, which is regarded as the gold standard of testing the differentiation potential of the MSCs. The multipotential differentiation capacity makes the MSCs an attractive target for cell-based regenerative therapies [1-3]. Thus, Mst1 inhibition may improve the curative effect of mBMSCs on PAH.

Although the clinical trial data provide evidence of the extraordinary safety profile of MSC therapy, scholarly literature has presented several reviews of the role of MSCs in the tumorigenic progression [2628]. Furthermore, anoikis resistance can protect tumor cells against mechanical stress-induced cell death [29]. Therefore, the improved anoikis resistance of MSCs may promote the initiation of tumor. In the present study, anoikis of mBMSCs was suppressed by Mst1 inhibition, while nude mouse tumorigenicity assay may demonstrate the safety profile of the administration of mBMSC/sh-Mst1.

Many experimental data have demonstrated that the deregulation of ITG and changes in their expression profile can contribute to several cellular processes, including cell survival, adhesion, and proliferation. Moreover, as a switch, by changing the ITG expression, cells could overcome anoikis [30]. ITGs are transmembrane $\alpha \beta$ heterodimers, and at least $18 \alpha$ and $8 \beta$ subunits are known. However, integrin heterodimers may differ in their ability to suppress apoptosis and survive under special circumstances [24]. For example, downregulation of ITGa5 $\beta 1$ can contribute to acquisition of anoikis resistance of human intestinal carcinoma cells. However, upregulation of ITGa5 31 leads to anoikis resistance by eliciting antiapoptotic signals, including the activation of the FAK $[5,24,31]$. In this study, ITGa5, and 
ITG $\beta 1$ were upregulated in the mBMSC/sh-Mst1 cultured in the cell-detachment condition. Moreover, ITGa5 and ITG $\beta 1$ were proposed to account for the Mst1 inhibition-induced anoikis resistance in the mBMSCs. Suppressing either ITGa5 or ITG $\beta 1$ by siRNA did not affect Mst1 expression, but Mst1 inhibition-induced anoikis resistance was abolished. These results suggested that ITGa5 $\beta 1$ acted as a downstream of Mst1 and a collaboration existed between ITGa5 and ITG $\beta 1$ involved in anoikis-resistant mBMSCs/sh-Mst1These phenomena suggested that suppression of Mst1 interacted with ITGa5b1 to support the anoikis resistance of the mBMSCs.

\section{Limitation}

Considering that mBMSCs were conferred anoikis resistance by the suppression of the Mst1 expression, further experiments need to be carried out. For example, we did not test the role of Mst1 overexpression in the mBMSCs cultured in the detached condition. We also did not exclude the possibility of additional factors that may promote anoikis resistance and the direct interaction between Mst1 and ITGa5 $\beta 1$.

\section{Conclusion}

Mst1 inhibition significantly improved cell survival, cell proliferation, and adhesion of the mBMSCs cultured in detached condition by activating the ITGa5 31 /FAK signal pathway. Moreover, Mst1 inhibition not only improved the multipotent capabilities of mBMSCs but also retained the safety profile of mBMSCs. Thus, Mst1 inhibition-based delivery may increase the therapeutic efficacy of MSCs, making them ideal candidates for clinical transplantation in PAH.

\section{Abbreviations}

mBMSC: mouse bone marrow mesenchymal stem cell;

PAH: pulmonary arterial hypertension;

ECM: Extracellular matrix;

Mst1: Mammalian Ste20-like kinase 1;

ITG: Integrin;

FAK: Focal adhesion kinase;

Poly-HEMA: poly-hydroxyethyl methacrylate;

CCK-8: Cell counting Kit 8;

TUNEL: Transferase-mediated dUTP nick-end labeling;

ALP: Alkaline phosphatase; 
PPARg: proliferator-activated receptor gamma;

FABP4: fatty acid binding protein 4;

\section{Declarations}

\section{Acknowledgments}

No applicable.

\section{Author Contributions}

Wancheng Yu contributed to the conception and design and manuscript writing; Tao Zhang contributed to conception and design, collection and assembly of data, data analysis and interpretation. Qian Zhang, Shang Wu, Haizhou Zhang performed the experiments; all authors participated in discussing, revising the manuscript, and approving the final manuscript.

\section{Funding}

This study was supported by a grant from the Natural Science Foundation of Shandong Province of China (No. ZR2020MH014).

\section{Availability of data and materials}

All data generated or analyzed during this study are included in this published article and its supplementary information files.

\section{Ethics approval and consent to participate}

This study was approved by the Research and Ethical Committee of the Shandong Provincial Hospital Affiliated to Shandong First Medical University, China, basing on the 1964 Helsinki Declaration and its later amendments.

\section{Consent for publication}

No applicable.

\section{Competing interests}

The authors indicated no potential conflicts of interest.

\section{References}

1. Bari E, Ferrarotti I, Torre ML, Corsico AG, Perteghella S (2019) Mesenchymal stem/stromal cell secretome for lung regeneration: The long way through "pharmaceuticalization" for the best 
formulation. J Control Release 309:11-24

2. Cruz FF, Rocco PRM (2020) The potential of mesenchymal stem cell therapy for chronic lung disease. Expert Rev Respir Med 14:31-39

3. Muhammad SA, Abbas AY, Saidu Y, Fakurazi S, Bilbis LS (2020) Therapeutic efficacy of mesenchymal stromal cells and secretome in pulmonary arterial hypertension: A systematic review and meta-analysis. Biochimie 168:156-168

4. Gomberg-Maitland M, Bull TM, Saggar R, Barst RJ, Elgazayerly A, Fleming TR, Grimminger F, Rainisio M, Stewart DJ, Stockbridge N, Ventura C, Ghofrani AH, Rubin LJ (2013) New trial designs and potential therapies for pulmonary artery hypertension. J Am Coll Cardiol 62:D82-D91

5. Paoli P, Giannoni E, Chiarugi P (2013) Anoikis molecular pathways and its role in cancer progression. Biochim Biophys Acta 1833:3481-3498

6. Zhang Z, Vuori K, Reed JC, Ruoslahti E (1995) The alpha 5 beta 1 integrin supports survival of cells on fibronectin and up-regulates Bcl-2 expression. Proc Natl Acad Sci U S A 92:6161-6165

7. Matter ML, Zhang Z, Nordstedt C, Ruoslahti E (1998) The alpha5beta1 integrin mediates elimination of amyloid-beta peptide and protects against apoptosis. J Cell Biol 141:1019-1030

8. Serakinci N, Guldberg P, Burns JS, Abdallah B, Schrodder H, Jensen T, Kassem M (2004) Adult human mesenchymal stem cell as a target for neoplastic transformation. Oncogene 23:5095-5098

9. Lang D, Lu MM, Huang L, Engleka KA, Zhang M, Chu EY, Lipner S, Skoultchi A, Millar SE, Epstein JA (2005) Pax3 functions at a nodal point in melanocyte stem cell differentiation. Nature 433:884-887

10. Hernanda PY, Pedroza-Gonzalez A, van der Laan LJ, Broker ME, Hoogduijn MJ, ljzermans JN, Bruno MJ, Janssen HL, Peppelenbosch MP, Pan Q (2013) Tumor promotion through the mesenchymal stem cell compartment in human hepatocellular carcinoma. Carcinogenesis 34:2330-2340

11. Robey TE, Saiget MK, Reinecke H, Murry CE (2008) Systems approaches to preventing transplanted cell death in cardiac repair. J Mol Cell Cardiol 45:567-581

12. Yu X, Cohen DM, Chen CS (2012) miR-125b Is an adhesion-regulated microRNA that protects mesenchymal stem cells from anoikis. Stem Cells 30:956-964

13. Lee S, Choi E, Cha MJ, Hwang KC, Cell adhesion and long-term survival of transplanted mesenchymal stem cells: a prerequisite for cell therapy, Oxid Med Cell Longev (2015) (2015) 632902

14. Song H, Chang W, Lim S, Seo HS, Shim CY, Park S, Yoo KJ, Kim BS, Min BH, Lee H, Jang Y, Chung N, Hwang KC (2007) Tissue transglutaminase is essential for integrin-mediated survival of bone marrow-derived mesenchymal stem cells. Stem Cells 25:1431-1438

15. Akimov SS, Krylov D, Fleischman LF, Belkin AM (2000) Tissue transglutaminase is an integrinbinding adhesion coreceptor for fibronectin. J Cell Biol 148:825-838

16. Zhang Q, Cheng X, Zhang H, Zhang T, Wang Z, Zhang W, Yu W (2020) Dissecting molecular mechanisms underlying $\mathrm{H} 2 \mathrm{O} 2$-induced apoptosis of mouse bone marrow mesenchymal stem cell: role of Mst1 inhibition. Stem Cell Res Ther 11:526 
17. Avruch J, Zhou D, Fitamant J, Bardeesy N, Mou F, Barrufet LR (2012) Protein kinases of the Hippo pathway: regulation and substrates. Semin Cell Dev Biol 23:770-784

18. Chou LY, Chen CH, Chuang SC, Cheng TL, Lin YH, Chou HC, Fu YC, Wang YH, Wang CZ, Discoidin Domain Receptor 1 Regulates Runx2 during Osteogenesis of Osteoblasts and Promotes Bone Ossification via Phosphorylation of p38, International journal of molecular sciences, 21 (2020)

19. Atashi F, Modarressi A, Pepper MS (2015) The role of reactive oxygen species in mesenchymal stem cell adipogenic and osteogenic differentiation: a review. Stem Cells Dev 24:1150-1163

20. Song H, Cha MJ, Song BW, Kim IK, Chang W, Lim S, Choi EJ, Ham O, Lee SY, Chung N, Jang Y, Hwang KC (2010) Reactive oxygen species inhibit adhesion of mesenchymal stem cells implanted into ischemic myocardium via interference of focal adhesion complex. Stem Cells 28:555-563

21. Taddei ML, Giannoni E, Fiaschi T, Chiarugi P (2012) Anoikis: an emerging hallmark in health and diseases. J Pathol 226:380-393

22. Gjorgieva D, Zaidman N, Bosnakovski D (2013) Mesenchymal stem cells for anti-cancer drug delivery. Recent Pat Anticancer Drug Discov 8:310-318

23. Porada CD, Almeida-Porada G (2010) Mesenchymal stem cells as therapeutics and vehicles for gene and drug delivery. Adv Drug Deliv Rev 62:1156-1166

24. Frisch SM, Vuori K, Ruoslahti E, Chan-Hui PY (1996) Control of adhesion-dependent cell survival by focal adhesion kinase. J Cell Biol 134:793-799

25. Wang S, Zhou L, Ling L, Meng X, Chu F, Zhang S, Zhou F (2020) The Crosstalk Between Hippo-YAP Pathway and Innate Immunity. Front Immunol 11:323

26. Galland S, Stamenkovic I (2020) Mesenchymal stromal cells in cancer: a review of their immunomodulatory functions and dual effects on tumor progression. J Pathol 250:555-572

27. Timaner M, Tsai KK, Shaked $Y(2020)$ The multifaceted role of mesenchymal stem cells in cancer. Semin Cancer Biol 60:225-237

28. Tang Q, Chen Q, Lai X, Liu S, Chen Y, Zheng Z, Xie Q, Maldonado M, Cai Z, Qin S, Ho G, Ma L (2013) Malignant transformation potentials of human umbilical cord mesenchymal stem cells both spontaneously and via 3-methycholanthrene induction. PLoS One 8:e81844

29. Strilic B, Offermanns S (2017) Intravascular Survival and Extravasation of Tumor Cells. Cancer Cell 32:282-293

30. Hynes RO (2009) The extracellular matrix: not just pretty fibrils. Science 326:1216-1219

31. Giancotti FG, Ruoslahti E (1999) Integrin signaling Science 285:1028-1032

\section{Figures}




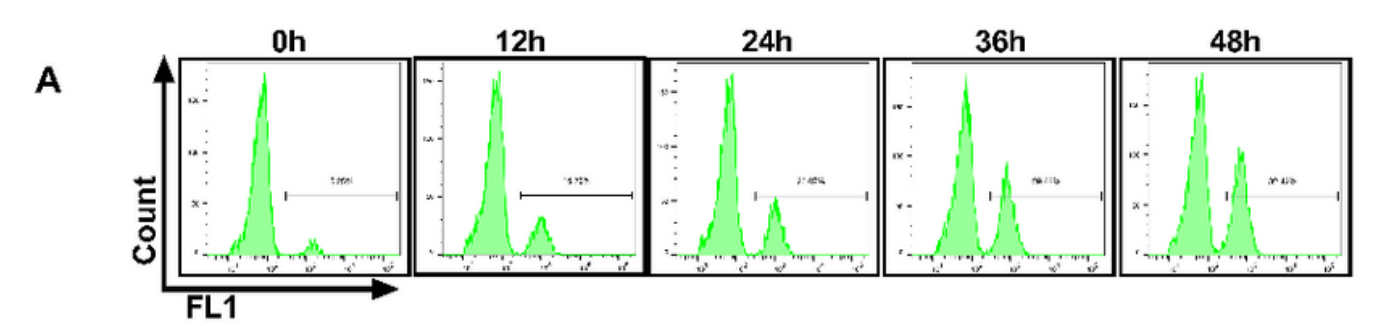

B

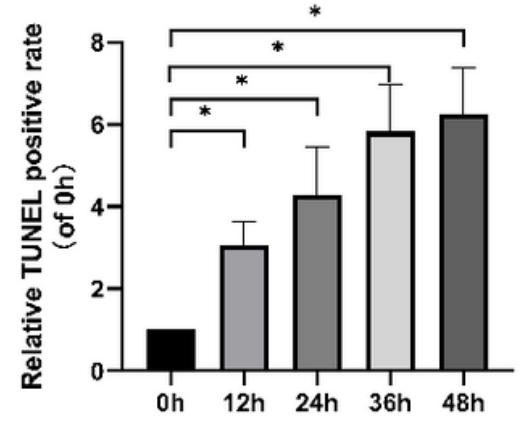

C

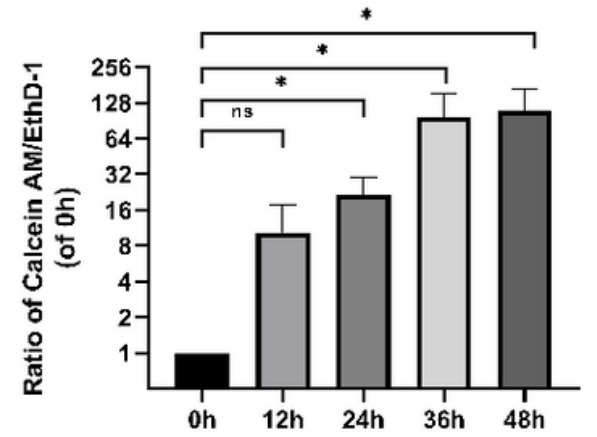

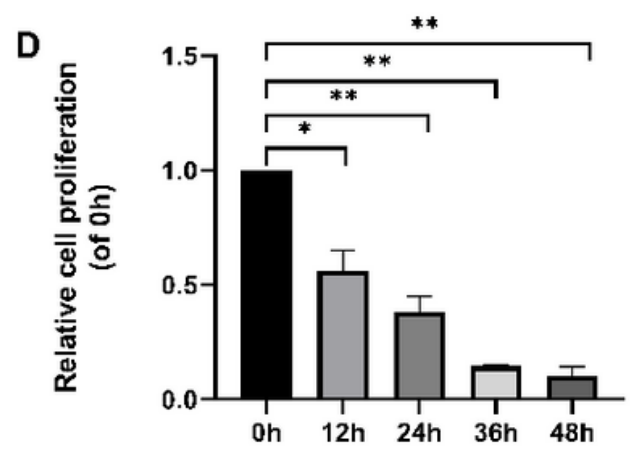
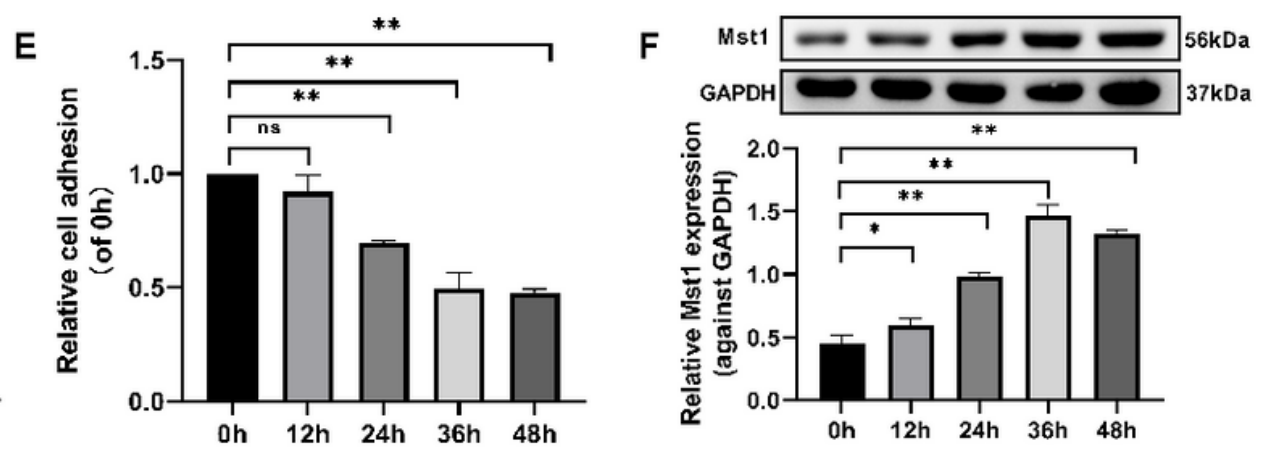

Figure 1

Poly-HEMA-treated mBMSCs exhibit susceptibility to anoikis. A. The ratio of TUNEL positive-cell (apoptotic cells) of mBMSCs in pre-coated poly-HEMA condition for $0 \mathrm{~h}, 12 \mathrm{~h}, 24 \mathrm{~h}, 36 \mathrm{~h}$ and $48 \mathrm{~h}$ using flow cytometry. B. Data expressed as fold of Oh groups. C. Anoikis Assay Kit was used to detect anoikis of mBMSCs cultured in pre-coated poly-HEMA condition for $0 \mathrm{~h}, 12 \mathrm{~h}, 24 \mathrm{~h}, 36 \mathrm{~h}$ and $48 \mathrm{~h}$. Quantitated with a fluorescence microplate reader and data expressed as fold of oh groups. D. Cell viability was measured in mBMSCs cultured in pre-coated poly-HEMA condition for $0 \mathrm{~h}, 12 \mathrm{~h}, 24 \mathrm{~h}, 36 \mathrm{~h}$ and $48 \mathrm{~h}$ by CCK-8. Data expressed as fold of Oh groups. F. Cell adhesion of mBMSCs was analyzed in mBMSCs in pre-coated poly-HEMA condition for $0 \mathrm{~h}, 12 \mathrm{~h}, 24 \mathrm{~h}, 36 \mathrm{~h}$ and $48 \mathrm{~h}$. Data expressed as fold of $0 \mathrm{~h}$ groups. G. Cell lysates from mBMSCs cultured in pre-coated poly-HEMA condition for $0 \mathrm{~h}, 12 \mathrm{~h}, 24 \mathrm{~h}, 36 \mathrm{~h}$ and $48 \mathrm{~h}$ were subjected to Western blot analysis for protein expression of Mst1. The expression of protein was expressed as fold of GAPDH. Values are mean \pm SD of three independent experiments in each case. ${ }^{* \star} p<0.01,{ }^{\star} p<0.05$, ncp $>0.05$. 

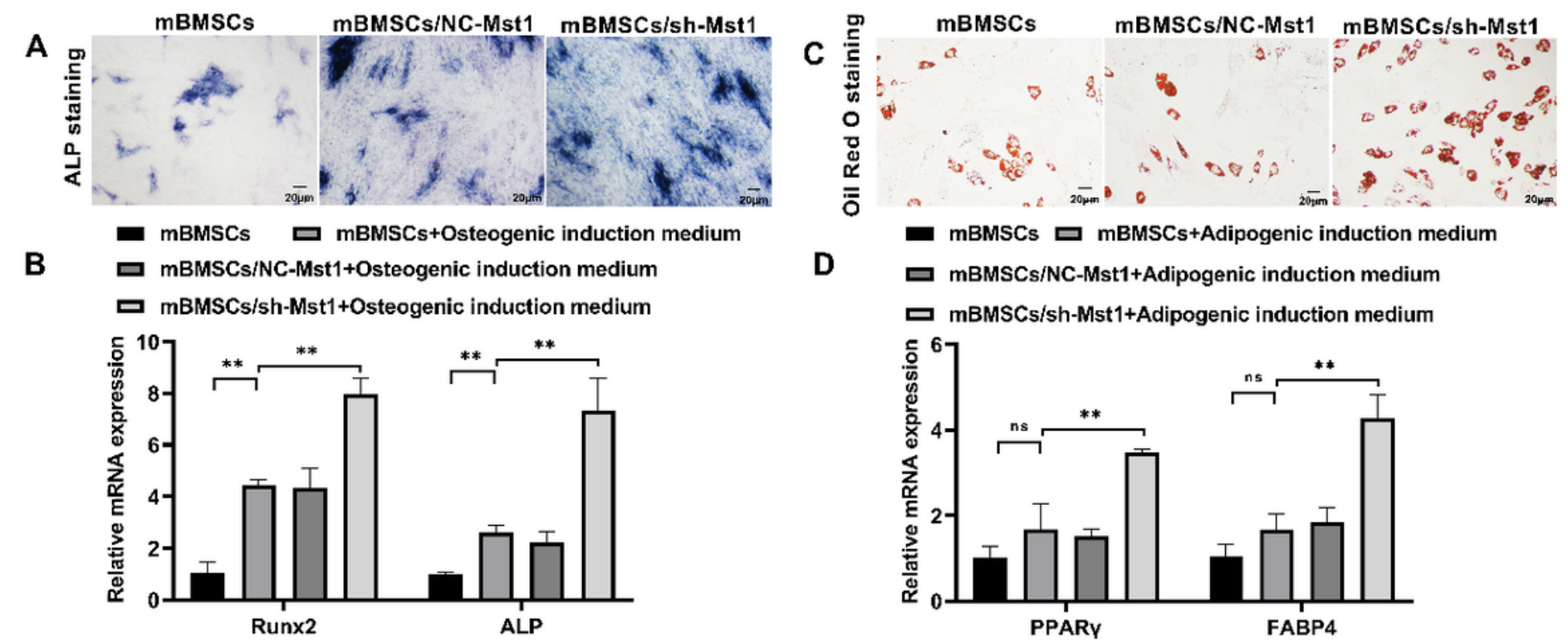

口 mBMSCs/NC-Mst1+Adipogenic induction medium

ㅁ mBMSCs/sh-Mst1+Adipogenic induction medium
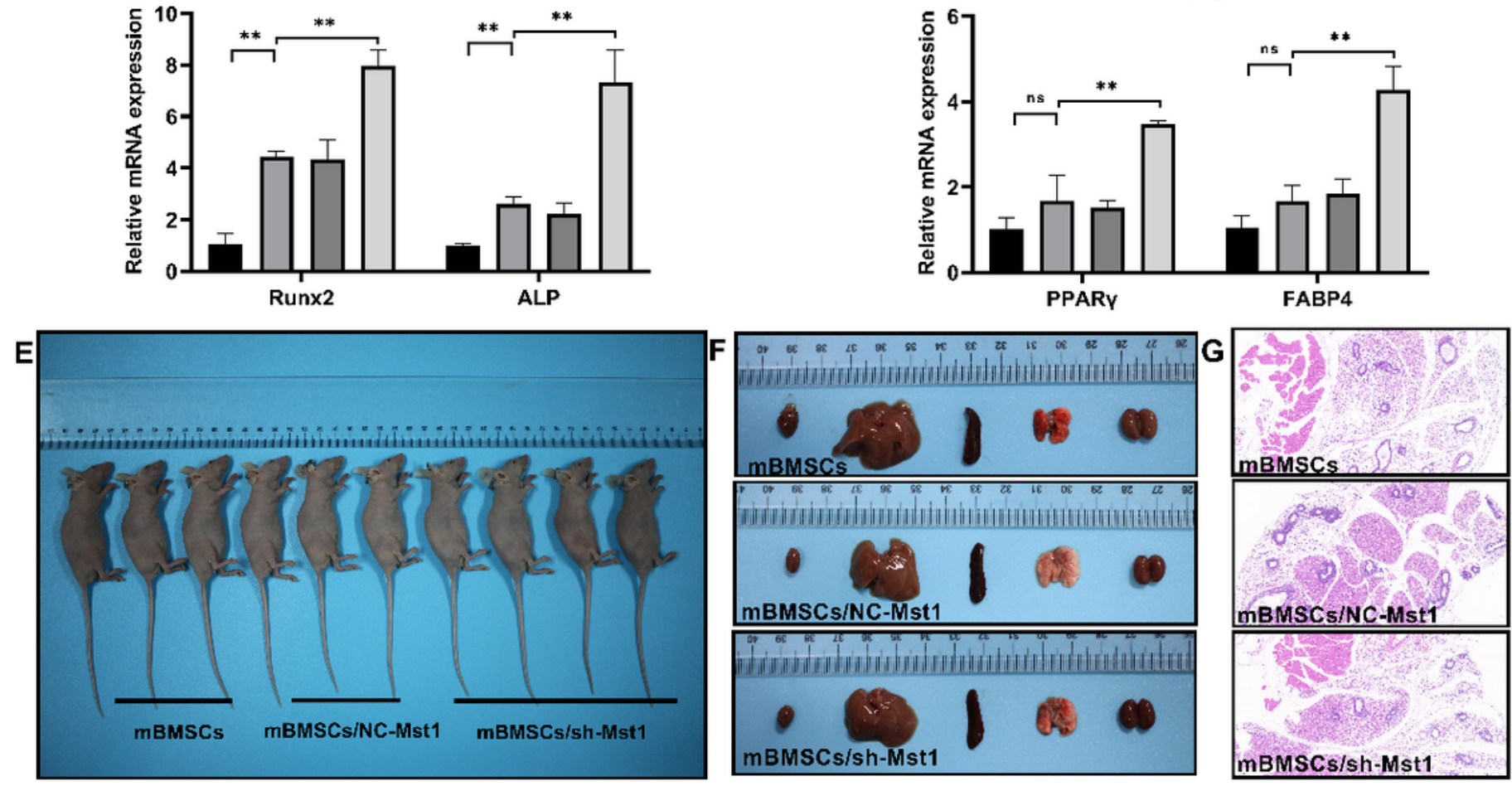

Figure 2

The osteoblastic and adipogenic differentiation potential of mBMSCs/sh-Mst1 and the safety profile of mBMSCs/sh-Mst1 administration. A. Representative images of ALP staining of mBMSCs after cultured in osteogenesis induction medium for 21 days. B. Representative images of Oil Red 0 staining of mBMSCs after cultured in adipogenesis induction medium for 21 days. C. qPCR analysis of the relative levels of Runx2 and ALP mRNA expression in mBMSCs cultured in adipogenesis induction medium for 21 days. D. qPCR analysis of the relative levels of PPAR $\triangle$ and FABP4 mRNA expression in mBMSCs cultured in adipogenesis induction medium for 21 days. E and F. Model was established in nude mice subcutaneously implanted with mBMSCs $(n=3)$, mBMSCs/NC-Mst1 $(n=3)$, mBMSCs/sh-Mst1 ( $n=4)$. Representative images of heart, liver, spleen, lung and kidney in each group after 60 days post-injection. G. Paraffin-embedded subcutaneous sections derived from cell-injection cites were stained with hematoxylin and eosin (H\&E). Data are mean \pm SD of three technical replicates. ${ }^{\star \star} p<0.01,{ }^{\star} p<0.05, n c p$ $>0.05$. 

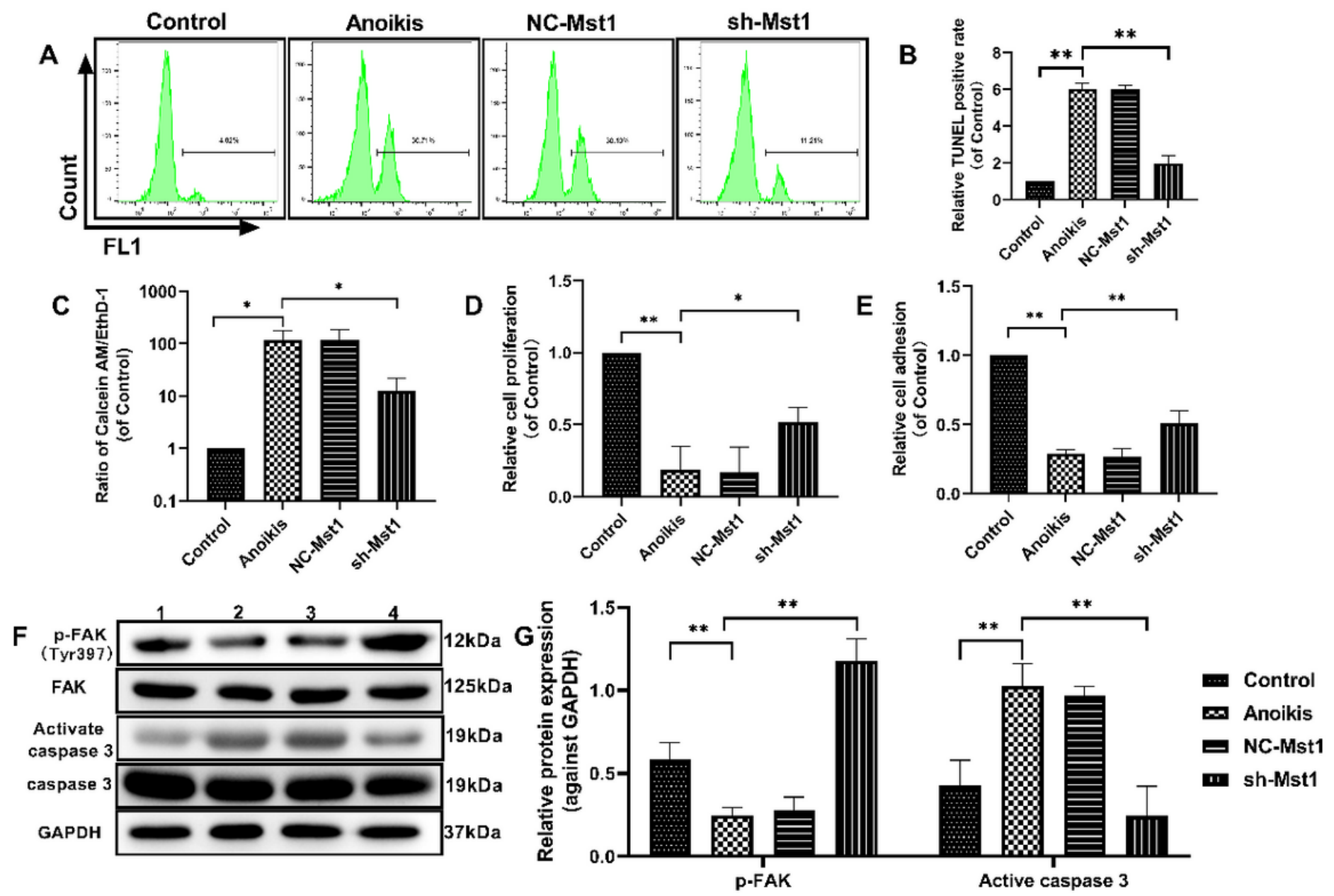

Figure 3

The effect of Mst1 inhibition on anoikis-mBMSCs. Cells were cultured in pre-coated poly-HEMA condition for $36 \mathrm{~h}$. A. The ratio of TUNEL positive-cell (apoptotic cells) analyzed by flow cytometry. B. The data were expressed as fold of control groups. C. Cells were stained with Calcein AM and EthD-1, then quantitated with a fluorescence microplate reader. The ratio was were normalized by control groups. D. Proliferation of mBMSCs as determined by CCK-8 assay and expressed as fold of control groups. E. Cell adhesion evaluated as fold of control groups. F and G. Expression of p-FAK (Tyr397) and activate caspase 3 as evaluated by Western blot analyses. GAPDH served as control. Values are mean \pm SD and experiments are completed in triplicate. ${ }^{* *} \mathrm{p}<0.01,{ }^{*} \mathrm{p}<0.05, \mathrm{ncp}>0.05$. 
A

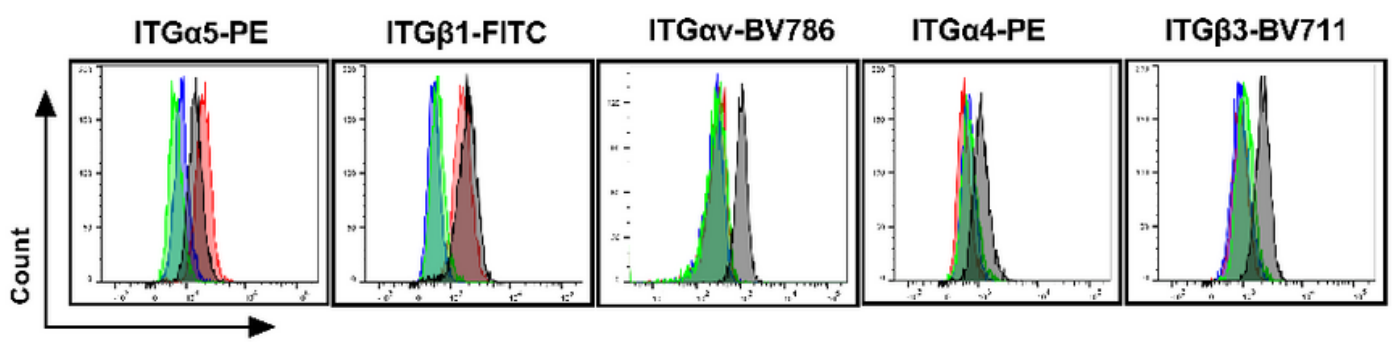

B
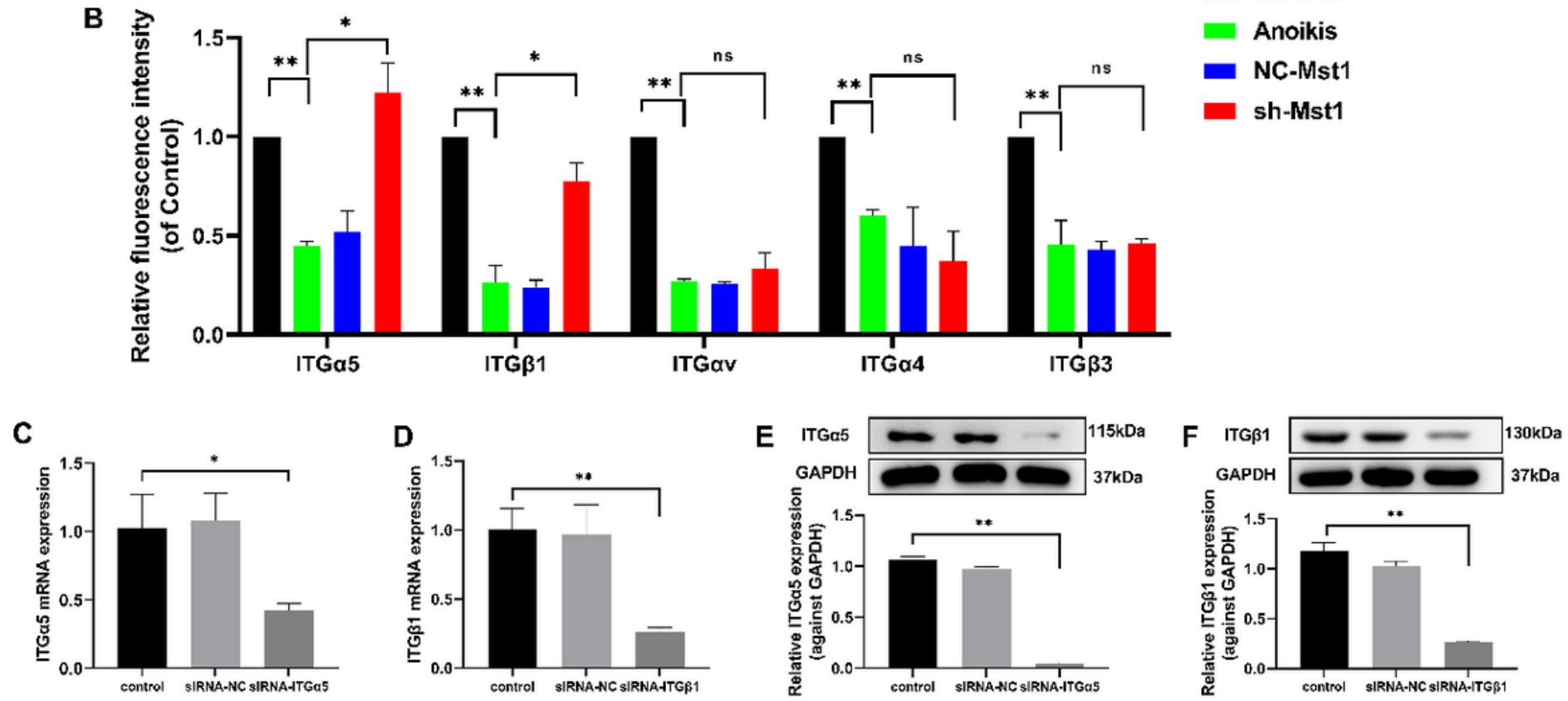

Figure 4

The change of ITG protein expression in mBMSCs/sh-Mst1 cultured in pre-coated poly-HEMA condition. Cells were cultured in pre-coated poly-HEMA condition for $36 \mathrm{~h}$. A. Flow cytometry analysis investigating the expression of the ITGa5, ITGav, ITGa4, ITG $\beta 1$, and ITG $\beta 3$, B. Data are expressed as fold of control groups. $C$ and D. mBM-MSC/sh-Mst1 was transfected with siRNA-NC, siRNA- ITGa5 and siRNA-ITG $\beta 1$, ITGa5 and ITG 11 expression levels were determined by PCR and Western blot analysis. GAPDH was used as the loading control. Values are expressed as mean $\pm S D, n=3$. ${ }^{\star *} p<0.01,{ }^{*} p<0.05, n c p>0.05$. 


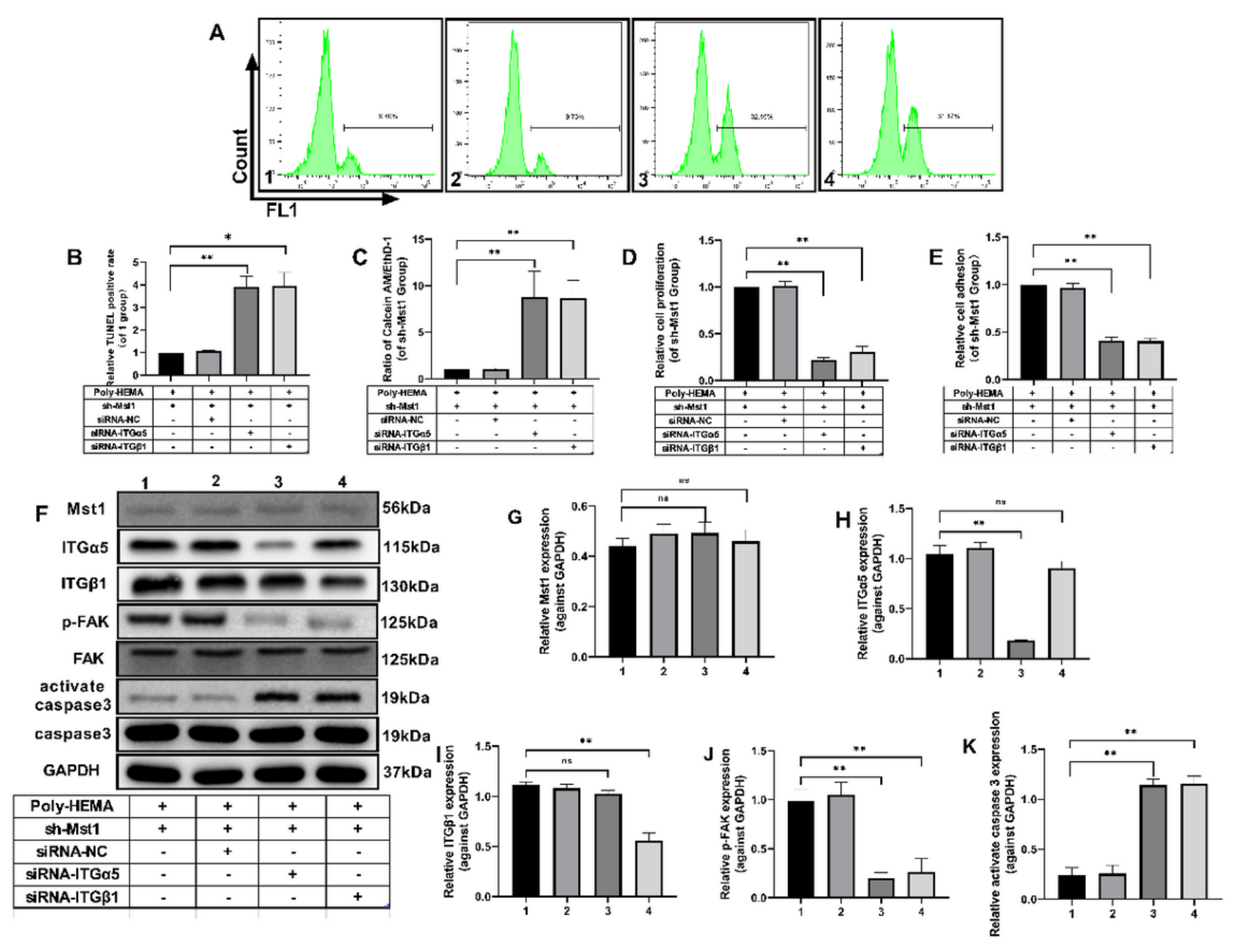

\section{Figure 5}

ITGa5 $31 /$ FAK signal pathway was activated in mBMSCs/sh-Mst1 cultured in pre-coated poly-HEMA condition. mBMSCs/sh-Mst1, mBMSCs/sh-Mst1 transfected with siRNA-NC, mBMSCs/sh-Mst1 transfected with siRNA- ITGa5, mBMSCs/sh-Mst1 transfected with siRNA-ITG $\beta 1$ were cultured in precoated poly-HEMA condition for $36 \mathrm{~h}$. A. The ratio of TUNEL positive-cell (apoptotic cells) analyzed by flow cytometry. B. The data were expressed as fold of control groups. C. Anoikis was also analyzed using Anoikis Assay Kit, and quantitated with a fluorescence microplate reader and normalized by sh-Mst1 groups. D. Cell proliferation assessed by CCK-8 assay and expressed as fold of sh-Mst1 groups. E. Cell adhesion expressed as fold of sh-Mst1 groups. F-K. Western blotting measures expression levels of Mst1, ITGa5, ITG 31 , p-FAK and activate caspase 3. GAPDH was used as the loading control. Values are expressed as mean \pm SD. Measurements were performed in three replicates. ${ }^{*} \mathrm{p} p<0.01,{ }^{\star} \mathrm{p}<0.05, \mathrm{ncp}$ $>0.05$. 1 group, Poly-HEMA treated mBMSCs/sh-Mst1; 2 group, Poly-HEMA treated mBMSCs/sh-Mst1 transfected with siRNA-NC; 3 group, Poly-HEMA treated mBMSCs/sh-Mst1 transfected with siRNA- ITGa5; 4 group, Poly-HEMA treated mBMSCs/sh-Mst1 transfected with siRNA- ITG $\beta 1$ 


\section{Supplementary Files}

This is a list of supplementary files associated with this preprint. Click to download.

- renamedecea1.docx 